\title{
Alpha-fetoprotein (AFP) and Chromosomal/Genetic Instability Disorders: Is AFP a Reporter Protein for DNA Damage-Sensing and Repair?
}

\author{
*Gerald J Mizejewski \\ New York State Department of Health, Wadsworth Center, USA
}

Submission: June 22, 2017; Published: June 27, 2017

"Correspondence Address: Gerald J Mizejewski, Division of Translational Medicine, New York State Department of Health, Wadsworth Center, P0 Box 509, Empire State Plaza Albany, NY 12201-0509, USA, Tel: (518) 486-5900; Fax: (518) 402-5002; Email: gerald.mizejewski@health.ny.gov

\begin{abstract}
Many cancer cells exhibit an unstable genome resulting from chromosomal aberrations and gene mutations in somatic cells which generate heterogeneous cell populations manifesting diseases such as cancer. Genomic/chromosomal instability can arise from non-repaired broken DNA along the chromosome or from loss of telomeric ends of chromosomes. Normally, DNA single or double-stranded breaks are repaired prior to the cells' entrance into the mitotic phase of the cell cycle. This progressive passage is overseen by DNA-damage sensors, checkpoint proteins, and kinase enzymes at each cyclic phase transition point. However, some cancer cells exhibit escape behaviors to evade checkpoint surveillance networks during cell cycle progression.
\end{abstract}

Examples of human diseases associated with DNA-associated disorders can be found in ataxia telangiectasia (AT), Fanconi's anemia (FA), immunodeficiency disorders, and others. Serum alpha-fetoprotein (AFP) levels have been reported as abnormal in these and other DNA/ chromosome instability disorders; in such situations, AFP appears to reflect the presence of impaired DNA damage and repair networks in certain cells. Although serum AFP presently serves as a biomarker in multiple disorders and diseases such as liver cancer and teratomas, the present discourse suggests that AFP might further serve as a reporter protein for the presence of DNA-damage sensing and repair networks in precancerous transforming cells.

Keywords: Alpha-fetoprotein; DNA repair; Immunodeficiencies; Cell cycle; BRCA proteins; Chromosome instability; Fanconi anemia; Breast cancer

Abbreviations: AT: Ataxia Telangiectasia; FA: Fanconi's Anemia; AFP: Serum Alpha-Fetoprotein; ATM: AT-mutated; ATR: AT-RAD-3 related protein

\section{Introduction}

\section{DNA Repair}

Cancer can be viewed as a condition of uncontrolled growth of cells as a result of chromosomal/genetic instabilities caused by DNA mutations. Gene mutations in somatic cells generate heterogeneous genomic populations which result in disease states such as cancer [1]. Many, if not most cancer cells, have an unstable genome comprised of aberrant pathways in DNA damage sensing and repair networks. DNA is constantly being damaged by metabolic products, ultraviolet rays, environmental chemicals (fertilizers, cosmetics, foodstuffs, etc.), free radicals, and other toxins. Interestingly, chemicals of the aldehyde (alcohol breakdown) class can also impede DNA repair [2]. Genomic instability arises either from non-repaired breaks in the DNA along the chromosome or from the loss of telomeres from the ends of chromosomes.

The DNA damage can encompass:

i. Broken and misshapen chromosomes;

ii. Broken chromatids; and

iii. Tri-radial and quadri-radial structures [3].

When cells continually divide or divide uncontrollably, some chromosomes are left with DNA double- or single-stranded breaks in middle or end chromosome regions. Normally, such breaks would be restored by the cells' DNA repair proteins; but if such genes are mutated in their repair mechanisms, situations result in which passage of corrupted DNA occurs through subsequent mitotic cell divisions. Such events lead to loss of gene function and a predisposition to various types of cancer. 


\section{DNA and the Cell Cycle}

One of the most remarkable discoveries aiding in understanding cancer within the past several decades has been elucidation of the regulatory mechanisms of cell division, growth, and proliferation involving a regulatory-type clock within cells referred to as the growth "cell cycle". The regulation of DNA damage-sensing out repair occurs through different phases of the cell cycle [4]. The activities of this built-in cellular clock occur through four phase periods, namely, the G1, S, G2, and mitosis (M) phases. Although the period in which cell division occurs is the mitotic phase, DNA repair must occur prior to the $\mathrm{M}$ phase so as to prevent propagating cells with corrupted DNA. In order for the cell to divide and grow, cell cycle-associated governing factors serve as regulators of DNA-damage sensing and repair to advance or halt (arrest) progression at each cell cycle phase [5].

As an overview, the various cell cycle regulators related to DNA repair encompass four classes; namely the

a. Cyclins;

b. Cyclin-dependent kinases (CdKs);

c. Checkpoint sensors; and

d. Proteosomal-associated ubiquitins including cullin proteins [6].

The Cullins are a family of hydrophobic proteins that serve as scaffolds and docking sites for ligases.

As addressed above, in order to preserve genomic stability mammalian cells employ cell cycle DNA surveillance stations termed cell cycle checkpoints to counteract and protect the genome from environmental/genotoxic insults. The DNA damage checkpoint networks consist of:

a) DNA damage sensors;

b) kinases;

c) ligases; and

d) Motor proteins.

Major kinases in DNA-repair include the phosphoinositide3-kinases such as ATM (ataxia telangiectasia (AT) mutated), ATR (AT-RAD-3 related protein), and DNA-PKcs (DNA-dependent protein kinase catalytic subunit) [7]. The substrates of these kinases mediate cell cycle arrest in the G1, S, and G2 phases of the DNA repair processes, as well as participate in cell death apoptotic pathways. Genetic mutations in the DNA damage response together with defective checkpoint components result in cancer, immune deficiency, and neurodegenerative disorders/ diseases. Unfortunately, cancer cells employ escape or evasion behaviors to bypass immune and checkpoint surveillance; these include both checkpoint arrest and subsequent recovery steps. Many of the DNA repair diseases such as AT and Fanconi anemia (FA) display immunodeficiency, neurological abnormalities, apoptosis, and high circulating serum levels of alpha-fetoprotein
(AFP). Interestingly, AFP serum levels appear to reflect over all dysfunction in cell cycle progression and DNA repair.

\section{DNA-Damage Sensing and Repair}

Since DNA damage can subject a person to future cancer susceptibility, mammalian cells contain complex networks of DNA damage-sensing and repair proteins. Human cancers can be the result of mutations that affect proteins in the DNA damage repair response in examples such as the AT-mutated (ATM) gene and the FA (FANC) genes [7]. These mutations are directly linked to a person's susceptibility to cancers such as leukemias and lymphomas. Reproduction-related cancers, exemplified by ovarian and breast, are associated with mutations in DNA damage response genes such as p53, BRCA1, and BRCA2. Patients bearing mutations in these genes, as in the ATM kinase, are some of the root causes of genomic instability in DNA repair disorders causing a variety of different cancers. As in the AT disorder, a mutated AT gene produces a misfolded kinase enzyme which can modify downstream targets such as p53 and BRCA1 [8]. When normal AT enzyme is activated, it promotes survival in host cells; in contrast, when AT genes are dysfunctional or mutated, it causes cells (including tumor cells) to become hypersensitive to radiation and/or chemotherapy. Thus, the mutated gene results in blockage of a kinase enzyme causing hypersensitivity to radiation which is readily observed in AT clinical patients [9].

When cellular DNA is damaged, cells rely on specific intracellular signaling pathways to stop cell division and repair the DNA before it is copied into subsequent cell progeny. Two such pathways are the ATM-CHK2 (checkpoint-2) and the ATRCHK1 (checkpoint-1) [10]. As stated above, many tumors also have defective p53 pathways (P53 is mutated or altered) which normally stop cell division when DNA is damaged [8]. To bypass this effect, alternate systems are employed and activated in tumor cells, such as the p38 MAPK/MK2 pathway. Tumors with a defective p53 pathway can become dependent solely on a pathway such as MK2. Other potential players in this arena may include MK3 and the ERK/M5 signaling modules. Since the MK2 pathway is also known to regulate inflammation and the immune response, potential MK inhibitors such as peptides, smallmolecule drugs, etc. show promise in treating tumor growth, and potentially, even inflammatory responses, autoimmune diseases, and immunodeficiencies.

\section{AFP and DNA-Associated Disorders}

DNA-related disorders often have, as their basis, dysfunctional networks that encompass cell processes associated with DNA synthesis, damage-sensing, and repair. Such human diseases or illnesses associated with AFP include various immunodeficiencies, anemia's, aneuploides, and gene/ chromosome instability disorders. Specific listings would encompass ataxia telangiectasia, Fanconi's anemia, severe combined immunodeficiency (with and without adenosine deaminase deficiency), acquired combined immunodeficiency 


\section{Cancer therapy \& Oncology International Journal}

(AIDS, HIV), fetal hemoglobin Bart's disease, erythropoietic porphyria (Gunther's disease), chromosomal abnormalities, and cerebellar ataxias. In all the above disorders, aberrant (increased or decreased) levels of serum AFP have been reported suggesting that AFP is somehow reflected in their pathogenesis.

Specific disorders of these groups, which involve AFP serum levels, are listed and described below. (Note: normal adult serum AFP is $8.0 \mathrm{ng} / \mathrm{ml}$ or less):

i. Adenosine deaminase deficiency (ADD) is involved in purine metabolism, nucleic acid turnover, and DNA synthesis. This enzyme-related group demonstrates DNA damage of cells of the immune system resulting in immune deficiencies similar to severe combined and general combined types. Elevated serum AFP levels are present in 33\% to $98 \%$ of patients with levels ranging from $30 \mathrm{ng} / \mathrm{ml}$ to nearly $500 \mathrm{ng} /$ $\mathrm{ml}$ depending on the type of immunodeficiency. In addition, maternal serum AFP in HIV-infected pregnant women have been reported to display elevated serum levels when compared to those from non-affected pregnant women [11].

ii. Fanconi's anemia is a genetic instability disorder in which patients' cells lack the capability to repair DNA breaks. Elevated serum AFP levels extend from $10 \mathrm{ng} / \mathrm{ml}$ to $70 \mathrm{ng} / \mathrm{ml}$ depending on the presenting ethnic (Turkish, French, etc.) variant and age of the patient. Furthermore, such patients have a susceptibility to breast cancer and other tumors [7].

iii. Fetal hemoglobin Bart's disease is a genetic instability disorder in which fetus's exhibit edema, hydrops, bone marrow failure, and moderate anemia. Elevated maternal serum AFP levels from such pregnant women range from $74 \mathrm{ng} / \mathrm{ml}$ to $171 \mathrm{ng} / \mathrm{ml}$ depending on gestational age of the fetus [12].

iv. Ataxia telangiectasia is another chromosomal instability disorder characterized by cerebellar ataxia, cutaneous telangiectasia, impaired immunocompetence, increased cell radio sensitivity, and susceptibility to lymphoid cancers. Elevations in juvenile and adult serum AFP levels extend from $30 \mathrm{ng} / \mathrm{ml}$ to $500 \mathrm{ng} / \mathrm{ml}$ and AFP levels increase with advancing age of the patient [11].

v. Erythropoietic porphyria (Gunther's disease) is a genetic disorder of pregnancy characterized by fetal ascites fluid accumulation, pericardial effusions, skin edema, and severe anemia. Elevated maternal serum AFP levels range from $200 \mathrm{ng} / \mathrm{ml}$ to $350 \mathrm{ng} / \mathrm{ml}$ depending on the gestational week of the pregnancy [12].

vi. Down syndrome (Trisomy-21) and Edward's Syndrome (Trisomy-18) are two chromosomal abnormalities which demonstrate growth delays, aberrant head and facial features, together with finger/hand defects and intellectual disabilities. Maternal serum AFP concentrations (expressed as multiple of medians) are reduced to levels of 0.4 to $0.9 \%$ that of gestational-age matched normal pregnancies [13].

vii. Spinocerebellar ataxias (oculomotor apraxia, AOA2) are a subtype of the AT disorder in which patients exhibit defects in single-stranded DNA repair responses and elevated levels of AFP. The gene mutation resides in the helicase domain of the Senataxin molecule in brain cerebellar cells. Elevated blood levels of AFP in AOA2 range from $20 \mathrm{ng} / \mathrm{ml}$ to $400 \mathrm{ug} /$ $\mathrm{ml}$ in 4- to 14-year old patients [14].

Overall, the disorders in patients mentioned above rarely exceed AFP levels of $500 \mathrm{ng} / \mathrm{ml}$ in contrast to liver disorders (hepatitis, cirrhosis, etc.) which range from $500 \mathrm{ng} / \mathrm{ml}$ to 3000

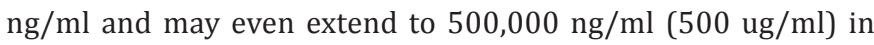
cases of advanced liver cancer [15]. Additional studies of AFP involvement in DNA damage and repair pathways have been recently reported. Microarray mRNA analyses of AFP-derived peptides have demonstrated that multiple cell cycle-associated proteins were down-regulated in MCF-7 breast cancer cells [16]. The cell-cycle proteins included checkpoint suppressor-1, cyclin-E, SKP2, DNA replication protein-ESCO2, and FANCD2. In other studies, intracytoplasmic AFP has been reported to promote hepatoma cell growth (in vitro) by binding and interacting with retinoic acid receptors, caspases, apoptosisinducing proteins, PI3K/AKT signaling components, Fn14 growth stimulators, PTEN, and GAAD153 (a growth arrest and DNA damage-inducible protein). These biological activities were all enacted by means of non-secreted AFP residing in the cell cytoplasm of hepatoma cells $[17,18]$.

\section{Conclusion}

DNA instability disorders are inexorably linked to cell cycle checkpoint proteins, kinases, and DNA-damage sensing and repair proteins. The cell cycle is the monitoring device to search out damaged DNA and arrest progression of the cell cycle in its progression toward mitotic cell division. Although the DNA damage sensing networks normally prevent corrupted DNA to transmit to daughter cells, mutations in the checkpoint proteins, cyclins, signal transducers, and kinases can interfere with these processes. The occurrence of aberrant AFP serum levels in adults and perinates have been reported which seem to reflect the presence of DNA-instability states of the genome (see above examples). At the cytoplasmic level, non-secreted AFP has also been shown to bind and interact with DNAassociated proteins, receptors, and transcription factors which influence the intracytoplasmic expression of such proteins. Although the presence of AFP in the serum of patients with DNA-instability disorders and the binding and protein-toprotein interaction of AFP with DNA repair proteins are known, [19] a subtle vocabulary of silence dominates this arena of the biomedical literature. Both serum- and cytoplasmic-derived AFP should be denoted as potential reporter proteins for genetic instability disorders involving DNA-damage sensing and repair. Such cognition might serve to alert clinicians, physicians, and researchers to probe for involvement and/or association of AFP 


\section{Cancer therapy \& Oncology International Journal}

in DNA instability disorders. Since AFP is already an established biomarker for birth defects, cancer, and hepatic disorders, physicians and clinical investigators should be made aware of the possible presence of DNA-impaired cells in patients with unexplained abnormal AFP levels.

\section{Acknowledgment}

The author extends his thanks and gratitude to Ms. Tracy Godfrey for her typing, corrections, revisions, and processing of this manuscript.

\section{Conflict of Interest}

The author declares that there are no known conflicts of interest in the preparation of this manuscript.

\section{References}

1. Chen L, Liu P, Evans TC Jr, Ettwiller LM (2017) DNA damage is a pervasive cause of sequencing errors, directly confounding variant identification. Science 355(6326): 752-756.

2. Tan SLW, Chadha S, Liu Y, Gabasova E, Perera D, et al. (2017) A Class of Environmental and Endogenous Toxins Induces BRCA2 Haploinsufficiency and Genome Instability. Cell 169(6): 1105-1118.

3. Clancy S (2008) DNA Damage \& Repair: Mechanisms for Maintaining DNA Integrity. Nature Education 1(1): 103.

4. Branzei D, Foiani M (2008) Regulation of DNA repair throughout the cell cycle. Nat Rev Mol Cell Biol 9(4): 297-308.

5. Bartek J, Lukas J (2007) DNA damage checkpoints: from initiation to recovery or adaptation. Curr Opin Cell Biol 19(2): 238-245.

6. Mizejewski GJ (2016) The alpha-fetoprotein (AFP) third domain: a search for AFP interaction sites of cell cycle proteins. Tumour Biol 37(9): 12697-12711.

7. Lakhi NA, Mizejewski GJ (2016) Alpha-fetoprotein and Fanconi Anemia: Relevance to DNA Repair and Breast Cancer Susceptibility. Fetal Pediatr Pathol 36(1): 49-61.

8. Peng L, Xu T, Long T, Zuo H (2016) Association Between BRCA Status and P53 Status in Breast Cancer: A Meta-Analysis. Med Sci Monit 22: 1939-1945.

9. Sinha RP, Hader DP (2002) UV-induced DNA damage and repair: a review. Photochem Photobiol Sci 1(4): 225-236.

10. Apostolou P, Papasotiriou I (2017) Current perspectives on CHEK2 mutations in breast cancer. Breast Cancer 9: 331-335.

11. Mizejewski GJ (2014) Alpha-fetoprotein as a biomarker in immunodeficiency diseases: relevance to ataxia telangectasia and related disorders. J Immunodeficiency \& Disorders 3: 1.

12. Mizejewski GJ (2016) Is alpha-fetoprotein a new biomarker for fetal, infant, and juvenile anemia? A commentary. J Hematology Research p. $10-12$.

13. Mizejewski GJ (2007) Physiology of alpha-fetoprotein as a biomarker for perinatal distress: relevance to adverse pregnancy outcome. Exp Biol Med (Maywood) 232(8): 993-1004.

14. Watanabe M, Sugai Y, Concannon P, Koenig M, Schmitt M, et al. (1998) Familial spinocerebellar ataxia with cerebellar atrophy, peripheral neuropathy, and elevated level of serum creatine kinase, gammaglobulin, and alpha-fetoprotein. Ann Neurol 44(2): 265-269.

15. Mizejewski GJ (2009) Alpha-fetoprotein (AFP)-derived peptides as epitopes for hepatoma immunotherapy: a commentary. Cancer Immunol Immunother 58(2): 159-170.

16. Mizejewski GJ (2011) Mechanism of Cancer Growth Suppression of Alpha-Fetoprotein Derived Growth Inhibitory Peptides (GIP): Comparison of GIP-34 versus GIP-8 (AFPep). Updates and Prospects. Cancers (Basel) 3(2): 2709-2733.

17. Li M, Li H, Li C, Wang S, Jiang W, et al. (2011) Alpha-fetoprotein: a new member of intracellular signal molecules in regulation of the PI3K/ AKT signaling in human hepatoma cell lines. Int J Cancer 128(3): 524532.

18. Mizejewski GJ (2015) Nonsecreted cytoplasmic alpha-fetoprotein a newly discovered role in intracellular signaling and regulation. An update and commentary. Tumour Biol 36(12): 9857-9864.

19. Mizejewski GJ (2017) The alpha-fetoprotein receptor binding fragment: Localization of third domain interaction sites of DNA repair proteins. Cancer therapy \& Oncology International Journal. In press.

\section{Your next submission with Juniper Publishers will reach you the below assets}

Commons Attribution 4.0 License

DOI: $10.19080 /$ CTOIJ.2017.05.555670 